\title{
Consideraciones para el examen clínico del síndrome de opérculo torácico neurogénico inespecífico
}

\author{
Considerations for clinical examination of nonspecific \\ neurogenic thoracic outlet syndrome
}

E. Pacheco y L. Rodríguez

Universidad San Sebastián, Facultad de Ciencias de la Salud. Valdivia, Chile

\section{ABSTRACT}

Thoracic outlet syndrome [SOT] is a controversial condition to this day, caused by compression of neurovascular structures in their passage between the neck and thorax. It is classified as vascular and neurogenic. If the electrophysiological tests are not positive it is subclassified as nonspecific neurogenic. This is between 95-98 \% of cases, but its diagnostic criteria are not yet clear. The main aspects to be taken into account during the clinical examination are reviewed to guide the diagnosis of patients with nonspecific neurogenic SOT, such as symptoms, triggers, differential diagnosis, assessment scales and psychosocial factors.

Key words: Thoracic outlet syndrome, neurovascular compression, cervicobrachialgia, differential diagnosis.

\section{RESUMEN}

El síndrome del opérculo torácico (SOT) es una condición causada por la compresión de las estructuras neurovasculares en su paso entre cuello y tórax. Se clasifica en vascular y neurogénico. Si las pruebas electrofisiológicas no son positivas, se subclasifica como neurogénico inespecífico. Este último constituye entre el 95 y el $98 \%$ de los casos, pero aún no están claros sus criterios diagnósticos. En el presente artículo se revisan los principales aspectos a tener en cuenta durante el examen clínico para guiar el diagnóstico de los pacientes con SOT neurogénico inespecífico, como sintomatología, factores desencadenantes, diagnóstico diferencial, escalas de valoración y factores psicosociales.

Palabras clave: Síndrome de opérculo torácico, compresión neurovascular, cervicobraquialgia, diagnóstico diferencial.

\section{INTRODUCCIÓN}

El síndrome de opérculo torácico (SOT) fue descrito como una entidad clínica por primera vez en 1956 por Peet, quien se refirió a este como "una compresión del paquete neurovascular de la extremidad superior en la zona del opérculo torácico" [1]. En la actualidad, este diagnóstico involucra cuadros clínicos con diferentes etiologías, que presentan en común el compromi- so de carácter compresivo de uno o más elementos neurovasculares en alguna parte de su recorrido por el opérculo torácico $[2,3]$.

Se estima que su incidencia es aproximadamente de un $8 \%$ en la población general (4), es extremadamente poco común en niños [5], tiene una edad de presentación que oscila entre los 20 y los 60 años de edad [6] y una mayor preferencia por el sexo femenino con una relación que va de 4:1 a 2:1 $[5,7]$. 
El SOT se caracteriza por presentar de manera aislada o combinada los siguientes síntomas: dolor, parestesias, debilidad y malestar. Con un agravamiento de los síntomas a la elevación de brazos, movimientos extremos de cabeza y cuello, cambios en la postura, posición escapular y un aumento nocturno de la sintomatología [8]. A causa del habitual solapamiento de estos síntomas, su diagnóstico, frente a otros cuadros de cuarto superior, se vuelve bastante complejo [9].

Un SOT puede presentar síntomas muy similares a: síndrome del túnel del carpo, tenosinovitis de Quervain, epicondilitis lateral, epitroclealgias, síndrome doloroso complejo regional tipo 1 y 2 , síndrome de Horner, enfermedad de Raynaud, cervicalgia mecánica, traumatismo del plexo braquial, trombosis venosa profunda de la extremidad superior, patología del manguito de los rotadores, inestabilidad glenohumeral, cefalea tensional y dolor orofacial, entre otros (9).

Mientras aún se debate si es una entidad clínica subdiagnosticada o sobrediagnosticada $(10,11)$, el SOT representa hasta hoy en día un desafío diagnóstico, tanto en el ámbito clínico como en el campo de la investigación.

\section{CLASIFICACIÓN Y FISIOPATOLOGÍA}

Históricamente, el SOT ha sido clasificado de acuerdo con su etiología en dos claras entidades $(7,9,12)$ : SOT vascular (SOTv) y SOT neurogénico [SOTn] (13). De acuerdo con Davinovic y cols. [4], solo un $2 \%$ de los pacientes diagnosticados con SOT son clasificados como SOTv, siendo el de origen venoso mucho más prevalente que el de tipo arterial $(14,16)$.

El $98 \%$ de los casos restantes son diagnosticados como SOTn, en donde el $1 \%$ es considerado verdadero y el otro $99 \%$ es clasificado como inespecífico $(13,17)$.

Finalmente, la literatura describe el llamado SOT mixto, que combina características clínicas de los cuadros anteriores (18).

Existen alteraciones congénitas, evidenciables por medio de pruebas de imagen, que normalmente se han asociado al diagnóstico de SOTn. Sin embargo, muchas de estas alteraciones permanecen asintomáticas por largos periodos de tiempo hasta que un evento traumático despierta la sintomatología (19). Esto queda de manifiesto en estudios en cadáveres que evidencian que más del 90 \% de la población pueden presentar anomalías anatómicas en la región opercular (20).

En las últimas décadas, y con el objetivo de mejorar el diagnóstico clínico, se ha subclasificado el SOT neurogénico como verdadero o inespecífico.

EI SOT neurogénico verdadero [SOTnv], presenta síntomas motores (mano de Gilliatt-Sumner), alteraciones neurofisiológicas cuantificables por medio de pruebas electrodiagnósticas y, generalmente, presencia de anormalidades anatómicas. Mientras que en el SOT neurogénico inespecífico [SOTni], no es posible encontrar una correlación estructural [21] ni confirmar su presencia mediante test electrodiagnósticos (22) o mediante la aplicación de test ortopédicos individuales, debido a su poca sensibilidad y especificidad (23), siendo el reporte de síntomas sensitivos intermitentes y dolor el principal motivo de consulta.
Es por esta razón que la aplicación de una exhaustiva recopilación de datos en la historia clínica, un acabado examen físico y un razonamiento clínico correcto, conforman las mejores herramientas diagnósticas [3,24].

En vista de lo anterior, queda en evidencia la necesidad de establecer criterios claros de evaluación y lineamientos de tratamiento para este grupo en particular.

\section{EXAMEN CLÍNICO}

Si bien la etiología de SOT es atribuida generalmente a patología ósea o alteraciones del tejido blando, estas no son requisito imperativo para su diagnóstico. En el caso de SOTn inespecífico, al no existir gold estándar, su pesquisa es fundamentalmente clínica y debe basarse en una detallada historia del caso y en la examinación objetiva y subjetiva del paciente (Tabla I). Sin embargo, es infrecuente lograr un nivel de certeza elevado. Con el fin de atenuar la incertidumbre diagnóstica, se presentan a continuación algunas de las principales consideraciones en el examen clínico.

Dentro del relato del paciente con SOTn inespecífico, la ubicación de la sintomatología es variable: parestesia en la extremidad superior (98\%), dolor cervical (88\%), dolor de trapecio (92\%), hombro y o brazo (88\%), dolor supraclavicular (76\%), dolor en el pecho (72 \%), dolor en región occipital (76\%), parestesias en los cinco dedos (58\%), solo el cuarto y quinto dedos (26\%) o en los primeros tres dedos (14\%) [25].

Dependiendo de la región del plexo que se encuentre más irritada, la clínica puede variar. Por ejemplo, si la compresión irrita más la porción superior del plexo [C5, C6, C7), los síntomas pueden expresarse como dolor en la cara anterior de la columna cervical, clavícula, mandíbula, oído y área mastoidea. Asimismo, pueden producirse irradiaciones a la cara y cefaleas ocasionales por anterior hacia la parte superior del tórax, por posterior en la región periescapular, y a través de la zona externa del brazo hacia el dorso del pulgar y el dedo índice. Por su parte, quienes presentan un mayor compromiso del plexo inferior (C8, T1) relatan principalmente síntomas en el borde cubital del brazo y la mano, y en ocasiones, combinado con síntomas en la región anterior del hombro y zona axilar [26]. A su vez, es relevante identificar si los síntomas se presentan en la actividad o durante la noche.

Un grupo de pacientes reporta despertar por parestesias en la extremidad, o bien sentirse inquietos, sin la posibilidad conciliar el sueño, viéndose obligados a cambiar repetidamente la postura al dormir. Este grupo de pacientes son conocidos como "releasers" [26]. Generalmente presentan brazos grandes y pesados, ya sea por tejido adiposo o masa muscular, con hombros redondeados y descendidos, escápulas en aducción/ retracción y un característico cuello alargado. Normalmente este grupo tiende a presentar Test de Cyriax positivo durante la examinación.

El mecanismo subyacente a este comportamiento es el compromiso nutricional del plexo braquial mediante la compresión o la tracción del paquete vasculonervioso. El cese de esta afectación mecánica durante la noche genera la reperfusión del plexo y producto de ello la aparición de las parestesias. Cabe mencionar que es 
TABLA I

CUADRO COMPARATIVO SINTOMATOLÓGICO

\begin{tabular}{|c|c|c|c|c|c|}
\hline \multirow{2}{*}{ Subtipo } & \multicolumn{2}{|c|}{ SOT vascular } & \multicolumn{2}{|c|}{ SOT neurogénico } & \multirow{2}{*}{ SOT traumático } \\
\hline & Venoso & Arterial & Verdadero & Inespecífico & \\
\hline $\begin{array}{l}\text { Signos y } \\
\text { sintomas }\end{array}$ & $\begin{array}{l}\text { Usualmente } \\
\text { en hombres } \\
\text { jóvenes } \\
\text { Historia de } \\
\text { deportes de } \\
\text { lanzamiento y de } \\
\text { alta intensidad } \\
\text { Usualmente } \\
\text { el edema es } \\
\text { unilateral } \\
\text { Dolor } \\
\text { Cianosis } \\
\text { Fatigabilidad } \\
\text { Sensación } \\
\text { de rigidez o } \\
\text { pesadez } \\
\text { Parestesia en } \\
\text { mano } \\
\text { Congestión } \\
\text { venosa con } \\
\text { colateralización } \\
\text { de vasos } \\
\text { periféricos } \\
\text { Trombosis } \\
\text { vena axilar o } \\
\text { subclavia } \\
\text { Embolia } \\
\text { pulmonar } \\
\text { parestesia } \\
\text { Usualmente } \\
\text { asociado a } \\
\text { variantes } \\
\text { anatómicas } \\
\text { Usualmente } \\
\text { asociado a } \\
\text { variantes } \\
\text { anatómicas }\end{array}$ & $\begin{array}{l}\text { Usualmente en } \\
\text { adultos jóvenes } \\
\text { Historia de } \\
\text { ejercicio } \\
\text { vigoroso } \\
\text { Dolor } \\
\text { espontáneo en } \\
\text { la mano } \\
\text { El dolor en } \\
\text { el hombro } \\
\text { o columna } \\
\text { cervical es poco } \\
\text { frecuente } \\
\text { Isquemia en } \\
\text { extremidad } \\
\text { superior } \\
\text { Embolización } \\
\text { arterial múltiple } \\
\text { Claudicación } \\
\text { Cianosis } \\
\text { Cambios } \\
\text { vasomotores } \\
\text { Gangrena digital } \\
\text { Ausencia o } \\
\text { disminución del } \\
\text { pulso arterial } \\
\text { Parestesias por } \\
\text { isquemia } \\
\text { Calambres } \\
\text { musculares } \\
\text { Intolerancia al } \\
\text { frío o calor } \\
\text { Usualmente } \\
\text { asociado a } \\
\text { variantes } \\
\text { anatómicas }\end{array}$ & $\begin{array}{l}\text { Predominio raíces } \\
\text { C5/6/7 } \\
\text { Cambios } \\
\text { sensoriales en } \\
\text { los primeros tres } \\
\text { dedos } \\
\text { Entumecimiento } \\
\text { en la mejilla, } \\
\text { lóbulo de la oreja, } \\
\text { brazo, cara lateral } \\
\text { del codo } \\
\text { Dolor cervical, } \\
\text { pecho } \\
\text { (pseudoangina), } \\
\text { hombro, brazo, } \\
\text { cara, mandíbula, } \\
\text { sienes, pabellón } \\
\text { auditivo y cefaleas } \\
\text { occipitales } \\
\text { Mareos, vértigo y } \\
\text { visión borrosa } \\
\text { Predominio raíces } \\
\text { C7/8/T1 } \\
\text { Cambios } \\
\text { sensoriales en } \\
\text { los dedos cuarto } \\
\text { y quinto, pérdida } \\
\text { de sensibilidad en } \\
\text { cara medial del } \\
\text { codo } \\
\text { Dolor y parestesia } \\
\text { cara medial del } \\
\text { codo, antebrazo y } \\
\text { meñique } \\
\text { Debilidad mano, } \\
\text { pérdida de } \\
\text { dixteria, atrofia } \\
\text { eminencia tenar e } \\
\text { hipotenar } \\
\text { Usualmente } \\
\text { asociado a } \\
\text { variantes } \\
\text { anatómicas }\end{array}$ & $\begin{array}{l}\text { Síntomas con } \\
\text { predominio } \\
\text { neurológico, } \\
\text { intermitentes y } \\
\text { transitorios } \\
\text { Parestesia } \\
\text { en dedos con } \\
\text { distribución variable } \\
\text { Sensación de fatiga } \\
\text { o debilidad en la } \\
\text { mano o en el brazo } \\
\text { Sensación de } \\
\text { rigidez, edema o } \\
\text { pérdida del control } \\
\text { motor } \\
\text { Dolor en columna } \\
\text { cervical y hombro, } \\
\text { codo espalda alta, } \\
\text { región pectoral, } \\
\text { supra y medio } \\
\text { escapular } \\
\text { Dolor cervical y } \\
\text { cefalea } \\
\text { Dolor luego de } \\
\text { posturas inusuales } \\
\text { o prolongadas } \\
\text { Rápida disminución } \\
\text { de los síntomas } \\
\text { después de un } \\
\text { cambio de postura, } \\
\text { usualmente en la } \\
\text { posición contraria } \\
\\
\text { Aumento de los } \\
\text { síntomas al final } \\
\text { del día } \\
\text { Pobre respuesta } \\
\text { a medicamentos } \\
\text { antinflamatorios } \\
\text { Ausencia de } \\
\text { historia de trauma }\end{array}$ & $\begin{array}{l}\text { Historia reciente } \\
\text { o antigua de } \\
\text { accidente } \\
\text { automovilístico } \\
\text { Síntomas con } \\
\text { predominio } \\
\text { neurológico, } \\
\text { intermitentes y } \\
\text { transitorios } \\
\text { Parestesia } \\
\text { en dedos con } \\
\text { distribución } \\
\text { variable } \\
\text { Sensación de } \\
\text { fatiga o debilidad } \\
\text { en la mano o en el } \\
\text { brazo } \\
\text { Sensación de } \\
\text { rigidez, edema o } \\
\text { pérdida del control } \\
\text { motor } \\
\text { Dolor en columna } \\
\text { cervical y hombro, } \\
\text { codo espalda alta, } \\
\text { región pectoral, } \\
\text { supra y medio } \\
\text { escapular } \\
\text { Dolor cervical y } \\
\text { cefalea } \\
\text { Dolor después de } \\
\text { posturas inusuales } \\
\text { o prolongadas } \\
\text { Rápida disminución } \\
\text { de los síntomas } \\
\text { después de un } \\
\text { cambio de postura } \\
\text { Puede estar } \\
\text { asociado a } \\
\text { variantes } \\
\text { anatómicas }\end{array}$ \\
\hline
\end{tabular}


un signo de buen pronóstico y guiará en gran medida el abordaje terapéutico [26].

Por otro lado, es posible caracterizar a un grupo de pacientes que experimentan síntomas especialmente durante el día, al realizar actividades con elevación de extremidades, mantener posturas prolongadas o efectuar maniobras que soliciten considerablemente la musculatura del cuarto superior y musculatura respiratoria accesoria. Se les identifica como "compressors". Es común identificar en la observación: postura de cabeza adelantada, hombros en protracción, escápulas en abducción y en algunos casos cifosis dorsal marcada. Usualmente este grupo de pacientes presentan Test de Ross positivo.

\section{DIAGNÓSTICO DIFERENCIAL}

Según los síntomas se puede clasificar la espondilosis cervical en: espondilosis con dolor cervical de origen multifactorial, radiculopatía y espondilosis cervical mielopática [27]. El flujograma de evaluación propuesto para patología cuarto superior se presenta en la Figura 1.

\section{Radiculopatía cervical}

La radiculopatía se caracteriza por presentar dolor, debilidad muscular, disminución de los reflejos tendinosos o atrofia muscular de cuarto superior. Los síntomas se originan por la compresión e inflamación del nervio espinal. La compresión puede ser generada por tejido discal blando con sintomatología aguda, tejido discal duro asociado a sintomatología crónica o, menos comúnmente, por hipertrofia facetaria [28]. Puede presentar un patrón compresivo (responde mejor a maniobras de tracción), inflamatorio (no presenta comportamiento mecánico claro) o mixto. Determinar el patrón contribuye a orientar el abordaje.

Los dos sitios de mayor aparición son la raíz C6 y C7. En caso de compromiso claro de C6 se observa: déficit del reflejo braquiorradial, debilidad del bíceps y dolor o parestesias en dirección al pulgar e índice. Por otro lado, la afección de la raíz C7 pueden ocasionar pérdida del reflejo tricipital, debilidad del tríceps y dolor o parestesias en el dedo medio. Es importante destacar que la presencia de estos síntomas radiculares definidos en los textos clásicos en muchos casos dista de la realidad clínica, por lo que es necesaria la aplicación de una completa valoración (29). En este sentido, los criterios descritos por Wainner resultan útiles para el descarte del cuadro: rotación cervical menor a $60^{\circ}$, Spurling test positivo, test de distracción positivo y test de tensión neural de la extremidad superior positivo. Con más de tres signos positivos la especificidad sube sobre el $90 \%$, siendo de gran utilidad clínica [30].

\section{Mielopatía cervical degenerativa}

La mielopatía cervical degenerativa, conocida también como mielopatía espondilótica cervical, es una patología neurológica producto de la compresión medu- lar en la columna cervical [31]. Es importante considerar su presencia en pacientes mayores de 50 años que presentan dolor y rigidez en la columna cervical o extremidades, pérdida del equilibrio, hormigueo, caídas frecuentes y/o incontinencia [32]. Cook y cols. (33) presentan unos criterios de predicción clínica para mielopatía. Estos son: 1) desviación de la marcha, 2] test de Hoffman positivo, 3] signo supinador invertido, 4) test de Babinsky positivo, 5) paciente mayor de 45 años. En la presencia de un signo positivo, el valor de la sensibilidad es de $94 \%$ y la especificidad de un $31 \%$. Sin embargo, en presencia de cuatro de los cinco signos la sensibilidad cambia a un $99 \%$ y la especificidad a un $100 \%$. Lo anterior lo transforma en una útil herramienta clínica.

\section{Dolor cervical inespecífico}

El dolor cervical inespecífico muchas veces es considerado un diagnóstico de exclusión, debido a que existen múltiples estructuras capaces de generar nocicepción en la columna cervical, variados factores de riesgo (sexo femenino, edad avanzada, altas demandas laborales, ser exfumador, poco apoyo social o laboral y antecedentes de trastornos cervicales o lumbares) y a una pobre asociación entre hallazgos imagenológicos de degeneración articular y dolor (34). Sin embargo, la sintomatología reportada puede expresarse como dolor axial en la columna cervical con limitación dolorosa en los movimientos de extensión rotación, dolor referido hacia uno de los dos hombros y región torácica superior, sensibilidad a la palpación, cefaleas y ausencia de signos neurológicos (35). Resulta interesante, entonces, considerarlo como un diagnóstico de exclusión en cuadros con compromiso cervical y de hombro.

\section{Dolor neuropático}

El descarte del dolor neuropático (DN) en el cuadro del paciente es fundamental durante la examinación, ya que este tipo de dolor se asocia a mayor severidad, requiere de una medicación específica y genera un impacto sustancial en la calidad de vida $[36,37]$. La identificación del DN requiere satisfacer principios y criterios neurológicos diagnósticos para demostrar la lesión o enfermedad que afecta al sistema somatosensorial. Esto puede ser complejo fuera del escenario de la Neurología, pero la literatura disponible ofrece herramientas para lograrlo. En ausencia de gold estándar para DN, la historia clínica, una distribución neuroanatómicamente plausible del dolor, termoalgesia positiva y la aplicación de la escala LANSS orientan hacia un desorden del sistema somatosensorial como causante del dolor en una topografía típica [38]. No siempre se tiene acceso a test diagnósticos objetivos para confirmar con certeza la lesión o enfermedad del sistema somatosensorial. Pero no es necesario. El nivel de certeza probable es suficiente para poner en marcha el tratamiento para DN. 


\section{Consulta dolor musculoesquelético} cuarto superior

\section{Screening psicosocial}

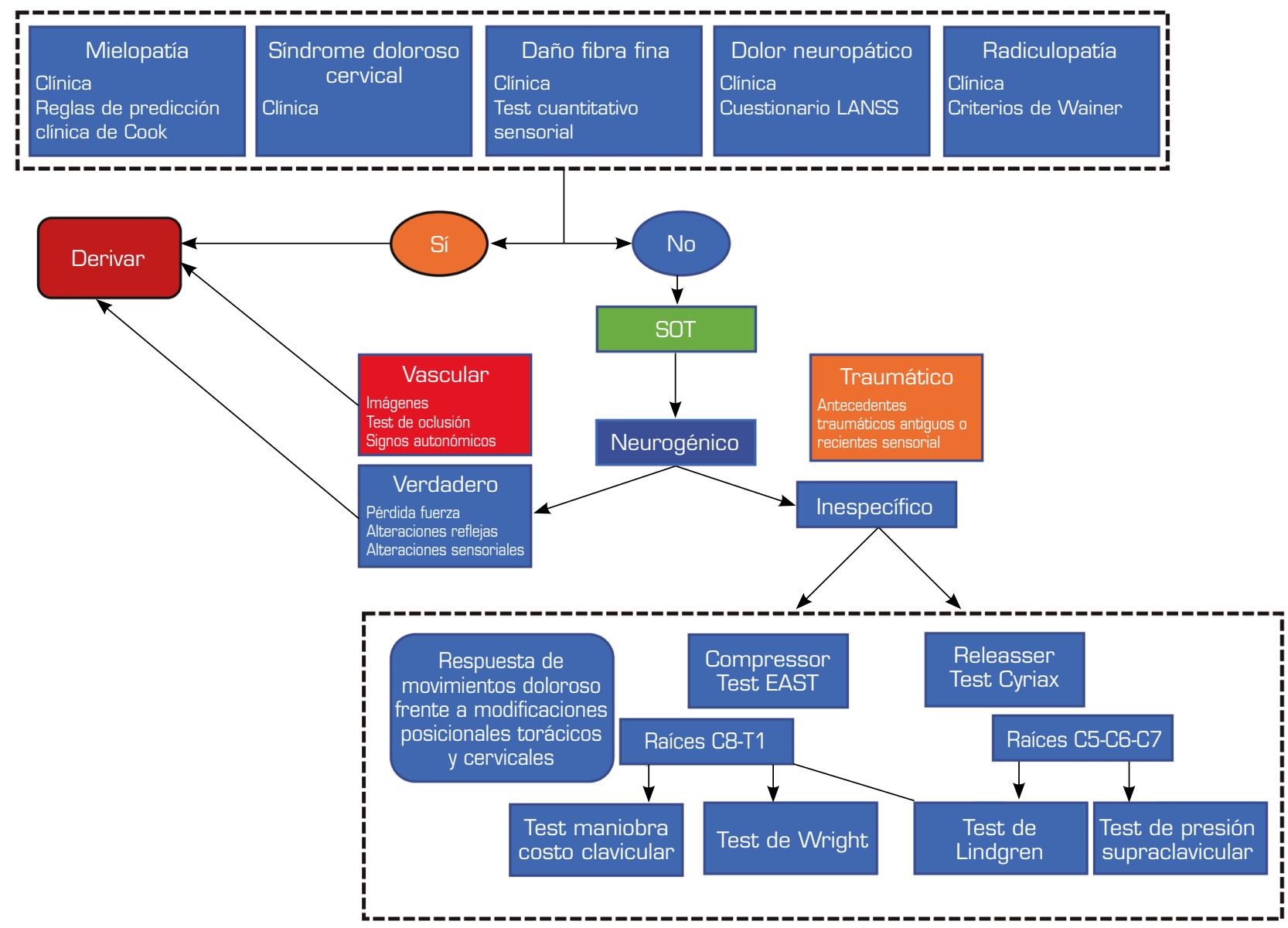

Test neurodinámicos específicos

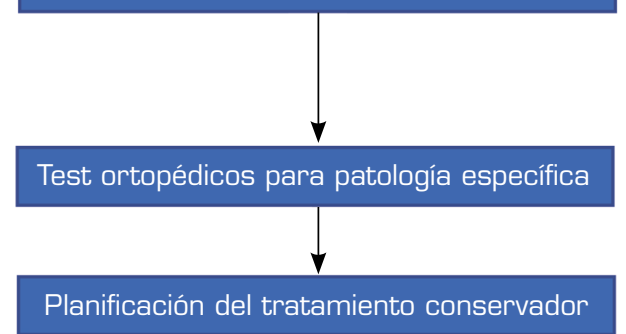

Fig. 1. Flujograma de evaluación propuesto para patología cuarto superior.

\section{Daño neural}

La confirmación del daño neural es a través de un estudio electrodiagnóstico y hallazgos clínicos concordantes con déficit neurológico (fuerza, tacto, reflejos). Sin embargo, no debemos olvidar que la electromiografía solo puede confirmar compromiso de fibras gruesas
(A-beta y motoras), que constituyen aproximadamente un $20 \%$ de un nervio periférico promedio. Para constatar el déficit sensitivo del $80 \%$ restante, compuesto por fibras finas (A-delta y $C$ ), se requiere de un test sensorial cuantitativo. Por consiguiente, aunque los síntomas se correlacionen fuertemente con el compromiso neural, es posible que estos test entreguen 
resultados negativos en presencia de SOTn inespecifico. Baselgia y cols. [39] concluyen que el $54 \%$ de las veces, un estudio de electromiografía positivo con test de tensión neural negativo indica compromiso, tanto de fibra gruesa como fina.

En algunas neuropatías por atrapamiento, como el síndrome del túnel carpiano, es posible evidenciar alteraciones de la función de fibras finas tanto distal como proximalmente $[40,41]$. Y aunque se solía pensar por experimentos en animales que esta compresión afectaba principalmente fibras gruesas $(42,43)$, estudios recientes sugieren que la compresión leve y progresiva, como ocurre en el SOTn inespecífico, afecta inicialmente a las fibras finas [44-46].

Resulta de suma importancia destacar que, ante este escenario, es útil complementar el examen con la evaluación de las fibras finas por la percepción de sensaciones punzantes agudas y umbral de temperatura mediante el test sensorial cuantitativo. Es más, Ridehalgh y Schmid [47), proponen que no es necesaria la biopsia de piel para objetivar la reducida inervación y disfunción de umbrales de presión y temperatura, sino que se puede usar en la clínica un mondadientes y una moneda (fría/caliente) para el mismo fin: simular un test sensorial. Para descartar la degeneración de fibra pequeña, tanto el umbral de detección de frío como el umbral de detección de calor deben ser negativos.

\section{MEDIDAS DE RESULTADO}

Un instrumento válido y confiable es la "Escala de función del miembro superior abreviada" (QuickDASH) [48]. Permite hacer una evaluación y registro rápido de la percepción de la limitación funcional de los pacientes con patologías de las extremidades superiores, con lo cual se pueden comparar cambios entre cada consulta médica y objetivar las variaciones en el compromiso funcional de estos. Es un método práctico para evaluar y realizar el seguimiento en pacientes ambulatorios. Además, se recomienda el Cervical Brachial Symptom Questionnaire (CBSQ) (49), que cuantifica la extensión de los síntomas relacionados con el SOT (dolor, fatiga, hinchazón, parestesia y entumecimiento) en la extremidad superior con ciertas actividades. Ambos instrumentos son complementarios.

\section{FACTORES PSICOSOCIALES}

Es esencial recordar que el dolor no es simplemente el producto final de un sistema de transmisión sensorial lineal, sino un proceso dinámico que implica interacciones continuas entre sistemas complejos, creencias, cultura, emociones, experiencias previas, entre otros.

Dentro de los factores afectivos asociados con el dolor, se incluyen el estrés y la ansiedad. Y dada la cronicidad e impacto de esta condición, no debe sorprender que un elevado número de pacientes puede cursar con trastorno de ansiedad. Hay amplia evidencia que demuestra que la ansiedad desmedida conlleva miedo al dolor, conductas de evitación, hiperreactividad muscular en respuesta al estrés y afrontamiento disfuncional $(50,51)$. Y esto tiene estrecha asociación con la cronificación del dolor. La edu- cación completa y empática al paciente es una estrategia eficaz para prevenir el desarrollo de estrés y ansiedad. Para objetivarla se recomienda usar la Escala State and Trait Anxiety Scale (STAl] [52).

Con el fin de maximizar las posibilidades de un resultado exitoso, es importante identificar los factores que ayudarían a determinar si es probable que un paciente se beneficie de una intervención o no. Los factores socioeconómicos asociados a la discapacidad laboral o los reclamos de indemnización de los trabajadores no diferencian los pacientes con SOT que responden al tratamiento de los que no. En una investigación se observaron varios factores frecuentes en pacientes resistentes al tratamiento quirúrgico: afecciones sensoriales que van más allá de los dermatomas del tronco inferior (42 vs. 10\%), debilidad que va más allá de los miotomas del tronco inferior (19 vs. $2 \%$ ), antecedentes de cirugía previa de cuello o extremidades superiores (50 vs. $17 \%$ ), comorbilidades de fibromialgia 0 sindrome de dolor regional complejo [81 vs. $12 \%$ ] y depresión [35 vs. 10\%] (49).

\section{CONCLUSIÓN}

El SOTn inespecífico es un posible diagnóstico al evaluar pacientes con síntomas de cuello y extremidad superior. A pesar de que no existen criterios diagnósticos específicos para determinar este síndrome, se puede establecer su existencia a través de una anamnesis clara que describa la sintomatología asociada, la aplicación de los instrumentos mencionados, y el descarte de otras causas potenciales para el examen clínico, y la eliminación de otras. La primera opción de atención clínica es el tratamiento conservador, que en la mayoría de los casos es suficiente para que el paciente recupere el funcionamiento normal.

\section{CONFLICTO DE INTERESES}

Los autores declaran no tener ningún conflicto de intereses.

\section{BIBLIOGRAFÍA}

1. Peet RM, Henriksen JD, Anderson TP, Martin GM. Thoracicoutlet syndrome: evaluation of a therapeutic exercise program. Proc Staff Meet Mayo Clin. 1956;31(9):281-7.

2. Levine N, Rigby B. Thoracic Outlet Syndrome: Biomechanical and Exercise Considerations. Healthcare. 2018;6(2):68. DOI: 10.3390/healthcare6020068.

3. Ferrante Mark A, Ferrante Nicole D. The Thoracic Outlet Syndromes: Part 1. Overview of the thoracic outlet syndromes and review of true neurogenic thoracic outlet syndrome. Muscle Nerve. 2017;55(6):782-93. D0I: 10.1002/ mus. 25536

4. Davidovic LB, Kostic DM, Jakovljevic NS, Kuzmanovic IL, Simic TM. Vascular thoracic outlet syndrome. World J Surg. 2003;27(5):545-50. DOI: 10.1007/s00268-003-6808-z.

5. Çağli K, Özçakar L, Beyazit M, Sirmali M. Thoracic outlet syndrome in an adolescent with bilateral bifid ribs. Clin Anat. 2006;19(6):558-60. DOl: 10.1002/ca.20280. 
6. Sanders RJ, Annest SJ, Goldson E. Neurogenic thoracic outlet and pectoralis minor syndromes in children. Vasc Endovascular Surg. 2013;47(5):335-41. DOI: $10.1177 / 1538574413481858$.

7. Gockel M, Vastamäki $M$, Alaranta $H$. Long-term results of primary scalenotomy in the treatment of thoracic outlet syndrome. J Hand Surg Br. 1994;19(2):229-33. DOI: 10.1016/0266-7681(94)90174-0.

8. Lindgren KA. Conservative treatment of thoracic outlet syndrome: A 2-year follow-up. Arch Phys Med Rehabil. 1997;78(4):373-8. DOI: 10.1016/S00039993(97)90228-8.

9. Watson LA, Pizzari T, Balster S. Thoracic outlet syndrome part 1: Clinical manifestations, differentiation and treatment pathways. Man Ther. 2009;14(6):586-95. DOI: 10.1016/j. math.2009.08.007.

10. Shukla PC, Carlton FB. Diagnosis of thoracic outlet syndrome in the emergency department. South Med J. 1996;89(2):2127. DOI: 10.1097/00007611-199602000-00011.

11. Wilbourn AJ. The thoracic outlet syndrome is overdiagnosed. Arch Neurol 1992;47(3):328-30. DOI: 10.1001/ archneur.1990.00530030106024.

12. Sharp WJ, Nowak LR, Zamani T, Kresowik TF, Hoballah JJ, Ballinger BA, et al. Long-term follow-up and patient satisfaction after surgery for thoracic outlet syndrome. Ann Vasc Surg. 2001;15(1):32-6. DOI: 10.1007/s100160010018.

13. Sanders RJ, Hammond SL, Rao NM. Thoracic outlet syndrome: A review. Neurologist. 2008;14(6):365-73. DOI: 10.1097/NRL.0b013e318176b98d.

14. Hussain MA, Aljabri B, Al-Omran M. Vascular Thoracic Outlet Syndrome. Semin Thorac Cardiovasc Surg. 2016;28(1):1517. DOI: 10.1053/J.SEMTCVS.2015.10.008.

15. Freischlag J, Orion K. Understanding Thoracic Outlet Syndrome. Scientifica (Cairo). 2014;2014:248163. DOI: 10.1155/2014/248163.

16. Hanna A, Bodden LON, Siebiger GRL. Neurogenic Thoracic Outlet Syndrome Caused by Vascular Compression of the Brachial Plexus: A Report of Two Cases. J Brachial Plex Peripher Nerve Inj. 2018;13(1):e1-e3. DOI: 10.1055/s0037-1607977.

17. Laulan J. Thoracic outlet syndromes. The so-called "neurogenic types." Hand Surg Rehabil. 2016;35(3):155-64. DOI: 10.1016/j.hansur.2016.01.007

18. Franklin GM. Work-Related Neurogenic Thoracic Outlet Syndrome. Diagnosis and Treatment. Phys Med Rehabil Clin N Am. 2015;26(3):551-61. DOI: 10.1016/j. pmr.2015.04.004.

19. Christo PJ, McGreevy K. Updated perspectives on neurogenic thoracic outlet syndrome. Curr Pain Headache Rep. 2011;15(1):14-21. DOI: 10.1007/s11916-010-0163-1.

20. Juvonen T, Satta J, Laitala P, Luukkonen K, Nissinen J. Anomalies at the thoracic outlet are frequent in the general population. Am J Surg. 1995;170(1):33-7. DOI: 10.1016/ s0002-9610(99)80248-7.

21. Sobey AVF, Grewal RP, Hutchison KJ, Urschel JD. Investigation of nonspecific neurogenic thoracic outlet syndrome. J Cardiovasc Surg (Torino). 1993;34(4):343-5.

22. Abe M, Katsuaki I, Nishida J. Diagnosis, treatment, and complications of thoracic outlet syndrome. Neurosurg Clin N Am 2001;12(2):295-309. DOI: 10.1007/s007760050075.

23. Dessureault-Dober I, Bronchti G, Bussières A. Diagnostic Accuracy of Clinical Tests for Neurogenic and Vascular Thoracic Outlet Syndrome: A Systematic Review. J Manipulative Physiol Ther. 2018;41(9):789-99. DOI: 10.1016/j. jmpt.2018.02.007.
24. Rayan GM. Thoracic outlet syndrome. J Shoulder Elbow Surg. 1998;7(4):440-51. DOI. 10.1016/S10582746(98)90042-8.

25. Sanders RJ, Hammond SL, Rao NM. Diagnosis of thoracic outlet syndrome. J Vasc Surg. 2007;46(3):601-4. DOl: 10.1016/j.jvs.2007.04.050.

26. Hooper TL, Denton J, McGalliard MK, Brismée J-M, Sizer PS. Thoracic outlet syndrome: a controversial clinical condition. Part 1: anatomy, and clinical examination/diagnosis. J Man Manip Ther. 2010;18(2):74-83. DOI: 10.1179/106 $698110 \times 12640740712734$.

27. Choi BW, Kim SS, Lee DH, Kim JW. Cervical radiculopathy combined with cervical myelopathy: prevalence and characteristics. Eur J Orthop Surg Traumatol. 2017;27(7):889-93. DOI: 10.1007/s00590-017-1972-2.

28. Voorhies RM. Cervical spondylosis: recognition, differential diagnosis, and management. Ochsner J. 2001;3(2):78-84.

29. Murphy DR, Hurwitz EL, Gerrard JK, Clary R. Pain patterns and descriptions in patients with radicular pain: Does the pain necessarily follow a specific dermatome? Chiropr Osteopat. 2009;17:1-9. DOI: 10.1186/1746-1340-17-9.

30. Wainner RS, Fritz JM, Irrgang JJ, Boninger ML, Delitto A, Allison S. Reliability and diagnostic accuracy of the clinical examination and patient self-report measures for cervical radiculopathy. Spine (Phila Pa 1976). 2003;28(1):52-62. DOl: 10.1097/00007632-200301010-00014.

31. Fehlings MG, Tetreault LA, Riew KD, Middleton JW, Aarabi B, Arnold PM, et al. A Clinical Practice Guideline for the Management of Patients With Degenerative Cervical Myelopathy: Recommendations for Patients With Mild, Moderate, and Severe Disease and Nonmyelopathic Patients With Evidence of Cord Compression. Glob Spine J. 2017;713 Suppl.): 70S-83S. DOI: 10.1177/2192568217701914.

32. Davies BM, Mowforth OD, Smith EK, Kotter MRN. Degenerative cervical myelopathy. BMJ. 2018;360(February):8-11. DOl: $10.1136 / \mathrm{bmj} . \mathrm{k} 186$.

33. Cook C, Brown C, Isaacs R, Roman M, Davis S, Richardson W. Clustered clinical findings for diagnosis of cervical spine myelopathy. J Man Manip Ther. 2010;18(4):175-80. DOl: 10.1179/106698110X12804993427045.

34. Blanpied PR, Gross AR, Elliott JM, Devaney LL, Clewley D, Walton DM, et al. Neck Pain: Revision 2017. J Orthop Sport Phys Ther. 2017;47(7):A1-A83. DOI: 10.2519/ jospt.2017.0302

35. Fukui S, Ohseto K, Shiotani M, Ohno K, Karasawa H, Naganuma $Y$, et al. Referred pain distribution of the cervical zygapophyseal joints and cervical dorsal rami. Pain. 1996;68(1):7983. DOI: 10.1016/S0304-3959(96)03173-9.

36. O'Connor AB. Neuropathic pain: quality-of-life impact, costs and cost effectiveness of therapy. Pharmacoeconomics. 2009;27(2):95-112. DOI: 10.2165/00019053200927020-00002.

37. Finnerup NB, Attal N, Haroutounian S, McNicol E, Baron R, Dworkin $\mathrm{RH}$, et al. Pharmacotherapy for neuropathic pain in adults: Systematic review, meta-analysis and updated NeuPSig recommendations. Lancet Neurol. 2015;14(2):162-73. DOI: 10.1016/S1474-4422(14)70251-0.

38. Wong CS, Hui GK, Chung EK, Wong SH. Diagnosis and management of neuropathic pain. Pain Manag. 2014;4(3):22131. DOI: 10.2217/pmt.14.7.

39. Baselgia LT, Bennett DL, Silbiger RM, Schmid AB. Negative Neurodynamic Tests Do Not Exclude Neural Dysfunction in Patients With Entrapment Neuropathies. Arch Phys Med Rehabil. 2017;98(3):480-4. DOI: 10.1016/j. apmr.2016.06.019. 
40. Schmid AB, Soon BT, Wasner G, Coppieters MW. Can widespread hypersensitivity in carpal tunnel syndrome be substantiated if neck and arm pain are absent? Eur $\mathrm{J}$ Pain. 2012;16(2):217-28. DOI: 10.1016/j.ejpain.2011.06.003.

41. Tampin B, Slater H, Hall T, Lee G, Briffa NK. Quantitative sensory testing somatosensory profiles in patients with cervical radiculopathy are distinct from those in patients with nonspecific neck-arm pain. Pain. 2012;153(12):2403-14. DOI: 10.1016/j.pain.2012.08.007.

42. Basbaum Al, Gautron M, Jazat F, Mayes M, Guilbaud G. The spectrum of fiber loss in a model of neuropathic pain in the rat: an electron microscopic study. Pain. 1991;47(3):359-7. DOl: 10.1016/0304-3959(91)90229-Q.

43. Dahlin LB, Shyu BC, Danielsen N, Andersson SA. Effects of nerve compression or ischaemia on conduction properties of myelinated and nonmyelinated nerve fibres. An experimental study in the rabbit common peroneal nerve. Acta Physiol Scand. 1989;136(1):97-105. DOI: 10.1111/j.17481716.1989.tb08634.x.

44. Schmid A, Coppieters M, Ruitenberg M, McLachlan E. Local and remote immune-mediated inflammation after mild peripheral nerve compression in rats. Schmid AB1, Coppieters MW, Ruitenberg MJ, McLachlan EM.No Title. J Neuropathol Exp Neurol. 2013;72(7):662-80. DOI: 10.1097/ NEN.Ob013e318298de5b.

45. Tamburin S, Cacciatori C, Praitano ML, Cazzarolli C, Foscato C, Fiaschi A, et al. Median nerve small- and large-fiber damage in carpal tunnel syndrome: $A$ quantitative sensory testing study. J Pain. 2011;12(2):205-12. DOI: 10.1016/j. jpain.2010.06.010.
46. Schmid AB, Bland JDP, Bhat MA, Bennett DLH. The relationship of nerve fibre pathology to sensory function in entrapment neuropathy. Brain. 2014;137(12):3186-99. DOI: 10.1093/brain/awu288.

47. Ridehalgh C, Sandy-Hindmarch OP, Schmid AB. Validity of Clinical Small-Fiber Sensory Testing to Detect Small-Nerve Fiber Degeneration. J Orthop Sport Phys Ther. 2018;48(10):76774. DOI: 10.2519/jospt.2018.8230.

48. García González GLA, Aguilar Sierra SF, Rodríguez Ricardo RMC. Validación de la versión en español de la escala de función del miembro superior abreviada: Quick Dash. Rev Colomb Ortop y Traumatol. 2018;32(4):215-9. DOI: 10.1016/j.rccot.2017.06.012.

49. Jordan SE, Ahn SS, Gelabert HA. Differentiation of thoracic outlet syndrome from treatment-resistant cervical brachial pain syndromes: Development and utilization of a questionnaire, clinical examination and ultrasound evaluation. Pain Physician. 2007;10(3):441-52.

50. Leeuw M, Goossens MEJB, Linton SJ, Crombez G, Boersma $\mathrm{K}$, Vlaeyen JWS. The fear-avoidance model of musculoskeletal pain: Current state of scientific evidence. J Behav Med. 2007;30(1):77-94. DOI: 10.1007/s10865-006-9085-0.

51. Vlaeyen JWS, Seelen HAM, Peters M, de Jong P, Aretz $E$, Beisiegel E, et al. Fear of movement/(re)injury and muscular reactivity in chronic low back pain patients: An experimental investigation. Pain. 1999;82(3):297-304. DOI: 10.1016/S0304-3959(99)00054-8.

52. Renzi DA. State-Trait Anxiety Inventory. Meas Eval Couns Dev. 1985;18(2):86-9. DOI: 10.1080/07481756.1985. 12022795. 